\title{
Individual commitment to a group effect: strengths and weaknesses of bovine embryo group culture
}

\author{
Eline Wydooghe, Leen Vandaele ${ }^{1}$, Sofie Piepers, Jeroen Dewulf, Etienne Van den Abbeel ${ }^{2}$, \\ Petra De Sutter ${ }^{2}$ and Ann Van Soom
}

Department of Reproduction, Obstetrics and Herd Health, Faculty of Veterinary Medicine, Ghent University, Eline Wydooghe, Salisburylaan 133, B-9820 Merelbeke, Belgium, ${ }^{1}$ Animal Sciences Unit, Institute for Agricultural and Fisheries Research (ILVO), B-9090 Melle, Belgium and ${ }^{2}$ Department of Reproductive Medicine, University Hospital, Ghent University, B-9000 Ghent, Belgium

Correspondence should be addressed to E Wydooghe; Email: Eline.Wydooghe@Ugent.be

\begin{abstract}
Recently, new culture devices such as Corral and Primo Vision dishes have been designed for the culture of human embryos to allow the combination of group culture plus follow-up of individual embryos. Bovine inseminated oocytes were allocated to Primo Vision dishes, Corral dishes, individual culture or classical group culture. Blastocyst development in Primo Vision dishes was similar to classical group culture (34.3 and $39.0 \%$ respectively), and better than Corral dishes or individual culture (28.9 and $28.5 \%$ respectively). In Primo Vision dishes, a higher number of 'slow' embryos developed to the blastocyst stage compared with their individually cultured counterparts, while no differences were observed for 'fast' embryos. 'Slow' embryos in a 'standard drop' had a higher chance of becoming a blastocyst compared with individual culture (OR: 2.3), whereas blastulation of 'fast' embryos was less efficient in a 'delayed drop' than in individual culture (OR: 0.3). The number of non-cleaved embryos in Primo Vision dishes did not negatively influence blastocyst development. Likewise, removing non-cleaved embryos (NC removed) and regrouping the cleaved embryos afterwards (ReGR) did not affect blastocyst development and quality compared with group culture in Primo Vision dishes (CTRL, 31.6\%, NC removed, 29.3\% and ReGR, 29.6\%). The experiments revealed that group culture of bovine embryos in Primo Vision dishes is superior to individual culture, primarily because of the higher blastocyst rate achieved by slow embryos. Non-cleaved or arrested embryos do not hamper the ability of co-cultured bovine embryos to reach the blastocyst stage in group culture.
\end{abstract}

Reproduction (2014) 148 519-529

\section{Introduction}

The merit of culturing embryos individually or in groups has been debated for almost 20 years. To date, contradictory reports have been published on the benefit of group culture over individual culture of human embryos (Rijnders \& Jansen 1999, Spyropoulou et al. 1999, Ebner et al. 2010, Rebollar-Lazaro \& Matson 2010, Tao et al. 2013), whereas many studies have described the benefits of group culture for bovine (Donnay et al. 1997, O'Doherty et al. 1997, Goovaerts et al. 2009, Wydooghe et al. 2014), mouse (Paria \& Dey 1990, Canseco et al. 1992, Lane \& Gardner 1992, Salahuddin et al. 1995), pig (Stokes et al. 2005) and cat (Spindler \& Wildt 2002) embryos. The main reason for this beneficial effect of group culture on animal embryo development has been ascribed to the secretion of autocrine factors by preimplantation embryos, which act upon the embryo itself or on neighbouring embryos (Paria \& Dey 1990, O'Neill 2008). In contrast, proponents of individual culture of human embryos argue that, regardless of the production of these embryotrophic factors, embryos in groups may be exposed to negative effects of dying or delayed embryos cultured in the same drop (Reed et al. 2011). In addition, another practical reason in favour of individual culture is the ability to follow morphological changes and development rates in individual embryos, which is of utmost importance in human IVF attempts.

Recently, new culture devices have been designed for human embryos, which allow the benefits of both group and individual culture approaches to be combined. One such device is the Corral dish (Sun IVF, Guelph, ON, Canada), which consists of two central wells, each divided into four quadrants and eight outer wells. The quadrant design allows the free passage of medium amongst the four quadrants, but the gaps between the quadrants are smaller than the diameter of an embryo, therefore individual monitoring is possible. Another type of culture device is based on the Well-of-theWell (WOW)-system, which was developed initially for zona-free bovine embryos (Vajta et al. 2000). In the 
bottom of a culture dish, microwells were created by fashioning small, round bottomed cylindrical wells and covering them with culture medium in order to create an optimum microenvironment for the embryo in each well (Vajta et al. 2000). Over the past decade, WOW dishes have been applied successfully to human IVF in combination with time-lapse photography. Two different models have been employed, the Embryo Slide dish, which allows the culture of individual embryos in microwells (Embryoscope), and the Primo Vision dish (Cryo Innovation Ltd, Budapest, Hungary and Vitrolife, Göteborg, Sweden) and Eeva dish (Auxogyn), both of which employ the WOW principle to permit group culture of embryos, each held in its own microwell while sharing the same drop of culture medium.

The aim of this study was to examine in more detail the effects of group culture using bovine embryos. Bovine embryos are very sensitive to suboptimal culture conditions and, in general, they develop less well in individual culture (Goovaerts et al. 2012). We compared the developmental outcome for bovine embryos cultured in these systems, with classical group culture (positive control: used routinely in bovine embryo culture (Fischer-Brown et al. 2002, Sagirkaya et al. 2007, Goovaerts et al. 2009, Wydooghe et al. 2014)) and individual culture (negative control). Furthermore, by using Primo Vision dishes we could investigate the influence of the developmental stage of the grouped embryos on the final developmental fate of any particular embryo cultured in a group compared with embryos cultured individually.

\section{Materials and methods}

\section{Media and reagents}

Basic Eagle's Medium amino acids, Minimal Essential Medium non-essential amino acids $(100 \times)$, TCM-199 medium, kanamycin and gentamycin were purchased from Life Technologies Europe and all other components were obtained from Sigma. All the media were filter-sterilised using a $0.22 \mu \mathrm{m}$ filter (Pall Corporation, Ann Arbor, MI, USA) before use.

\section{In vitro embryo production}

Bovine embryos were produced by routine in vitro methods described previously by Wydooghe et al. (2014). Briefly, cow ovaries were collected from a local slaughterhouse and processed within $2 \mathrm{~h}$. The ovaries were washed three times in warm physiological saline supplemented with kanamycin $(25 \mathrm{mg} / \mathrm{ml})$. The cumulus-oocyte complexes were aspirated from antral follicles between 4 and $8 \mathrm{~mm}$ diameter using an 18-gauge needle attached to a $10 \mathrm{ml}$ syringe. Oocytes with uniformly granulated cytoplasm and surrounded by more than three compact layers of cumulus cells were selected and these were matured in groups of 60 in $500 \mu \mathrm{l}$ modified bicarbonatebuffered TCM-199 supplemented with $20 \mathrm{ng} / \mathrm{ml}$ epidermal growth factor and $50 \mu \mathrm{g} / \mathrm{ml}$ gentamicin in $5 \% \mathrm{CO}_{2}$ in air for $22 \mathrm{~h}$ at $38.5^{\circ} \mathrm{C}$. Frozen-thawed bull spermatozoa were passed over a Percoll gradient (45 and 90\%; GE Healthcare Biosciences, Uppsala, Sweden) and then washed. The final concentration of $1 \times 10^{6} \mathrm{sperm} / \mathrm{ml}$ was adjusted using IVF-Tyrode's AlbuminPyruvate-Lactate (TALP), which consisted of bicarbonatebuffered Tyrode's solution, supplemented with BSA (Sigma A8806; $6 \mathrm{mg} / \mathrm{ml})$ and heparin $(25 \mu \mathrm{g} / \mathrm{ml})$. The matured oocytes were washed in $500 \mu \mathrm{l}$ of this IVF-TALP and incubated with spermatozoa for $21 \mathrm{~h}$. This procedure resulted in a fertilisation rate (two pronuclei seen) of $77.1 \%$ and a penetration rate (at least two pronuclei seen) of $83.6 \%$, evaluated after Hoechst staining in similar experiments (three replicates) as described by Thys et al. (2009). After fertilisation, surplus spermatozoa and cumulus cells were removed by vortexing and the inseminated oocytes were transferred to synthetic oviduct fluid supplemented with essential and non-essential amino acids (SOFaa) plus $0.4 \%$ BSA (Sigma A9647) and ITS $(5 \mu \mathrm{g} / \mathrm{ml}$ Insulin $+5 \mu \mathrm{g} / \mathrm{ml}$ transferrin $+5 \mathrm{ng} / \mathrm{ml}$ selenium). The number of inseminated oocytes per group and the volume of culture medium they were incubated in depended on the experimental set-up.

\section{Experiment 1: comparison of different group culture systems}

A total of 1572 bovine oocytes (four replicates) were randomly allocated to four experimental groups as detailed in Table 1. Two of the systems have been approved for use in human embryo culture (Primo Vision dish and Corral dish; Fig. 1B and $\mathrm{C}$ ) and the other two systems (group and individual cultures; Fig. 1A and D) had been tested previously in our laboratory (Wydooghe et al. 2014). It should be noted that in this experimental set-up, the differences in embryo density are uncontrolled and may be a significant confounder in the interpretation of the data.

\section{Experiment 2: influence of non-cleaved embryos in group culture}

After fertilisation, all inseminated oocytes $(n=629$, five replicates) were cultured in Primo Vision dishes. During the course of the experiments, the Primo Vision dish was modified from that designed for ten embryos to one designed for nine embryos arranged in a $3 \times 3$ raster. In Experiment 2, therefore, all inseminated oocytes were cultured in groups of nine, with each of them in one microwell, and immersed in $30 \mu \mathrm{l}$ SOF medium. At $45 \mathrm{~h}$ post insemination (hpi), embryo cleavage was

Table 1 Summary of the different culture systems used for the four experimental groups.

\begin{tabular}{lcccc}
\hline & Group & $\begin{array}{c}\text { Primo } \\
\text { Vision }\end{array}$ & Corral & Individual \\
\hline Drop size $(\mu \mathrm{l})$ & 50 & 30 & 120 & 20 \\
Embryos/drop & 25 & 10 & 4 & 1 \\
Embryo density & $1: 2$ & $1: 3$ & $1: 30$ & $1: 20$ \\
Inter-embryo $_{\quad}$ & $1-500$ & 680 & 4000 & - \\
\hline distance $(\mu \mathrm{m})^{\mathrm{a}}$ & & & & \\
\hline
\end{tabular}

${ }^{\mathrm{a}}$ Measured from centre-point to centre-point. 
assessed, and three experimental groups were created in which: i) no additional handling was performed (CTRL), ii) noncleaved embryos (NC) were removed and iii) non-cleaved embryos were removed and the cleaved embryos were grouped together (ReGR).

\section{Evaluation of embryo development}

At $45 \mathrm{hpi}$, embryo cleavage rate was evaluated as the percentage of inseminated oocytes that cleaved. The kinetics of cleavage was also assessed and embryos that had proceeded to the third cleavage division (five to eight cells) were classified as 'fast embryos' whereas those that had reached only the second cleavage division (two to four cells) were categorised as 'slow embryos' (Vandaele et al. 2007). The percentage of inseminated oocytes to reach the blastocyst stage of development were assessed at 168 and $192 \mathrm{hpi}$ and at the latter timepoint the percentage of blastocysts that had hatched from their zona pellucidae were also recorded.

\section{Evaluation of embryo quality}

At $192 \mathrm{hpi}$, all the blastocysts were fixed in $2 \%$ paraformaldehyde (w/v) for $20 \mathrm{~min}$ and subsequently subjected to differential apoptotic staining to assess the quality of their morphological development (Wydooghe et al. 2011a). This involved double immunofluorescent staining with firstly, CDX2, a transcription factor uniquely expressed in the trophectoderm (TE) cells, secondly, with active caspase-3 which plays a central role in all apoptotic pathways. This staining was further combined with a Hoechst nuclear stain. This method allowed simultaneous evaluation of three important parameters of embryo quality: i) the total cell number (TCN), ii) the proportion of cells of inner cell mass (ICM ratio) calculated by subtraction of the number of TE cells from the TCN, divided by the TCN and iii) the percentage of apoptotic cells (apoptotic cell ratio (ACR)). In addition, the absolute ICM-number (calculated as TCN-TE) and apoptotic cell number were also counted.

\section{Comparative efficiency of group vs individual culture methods}

Comparison of the embryos in Primo Vision culture dishes with those cultured individually in Experiment 1 gave valuable insights into the group culture phenomenon. Therefore, retrospectively, blastocyst development at $192 \mathrm{hpi}$ for those groups cultured in Primo Vision dishes vs those cultured individually was related to whether the embryos had been 'slow' (two to four cells) or 'fast' (five to eight cells) developers at $45 \mathrm{hpi}$, and what effect, if any, the number of neighbouring embryos may have had on the rates of blastocyst development of both 'fast' and 'slow' embryos. Similarly, did the stage of development of neighbouring embryos in Primo Vision dishes improve or impair blastocyst development. Finally, the effect upon the embryos of being cultured in a 'standard' or a 'delayed' drop was investigated, based on the developmental stages of the nine embryos in the group. The definition of 'standard' and 'delayed' drop in the Primo Vision culture at the different time points was based on the development of embryo in the control group (classical group culture; Table 2).

\section{Statistical analysis}

To analyse the data on embryo development in both experiments, a binary logistic regression model of IBM SPSS
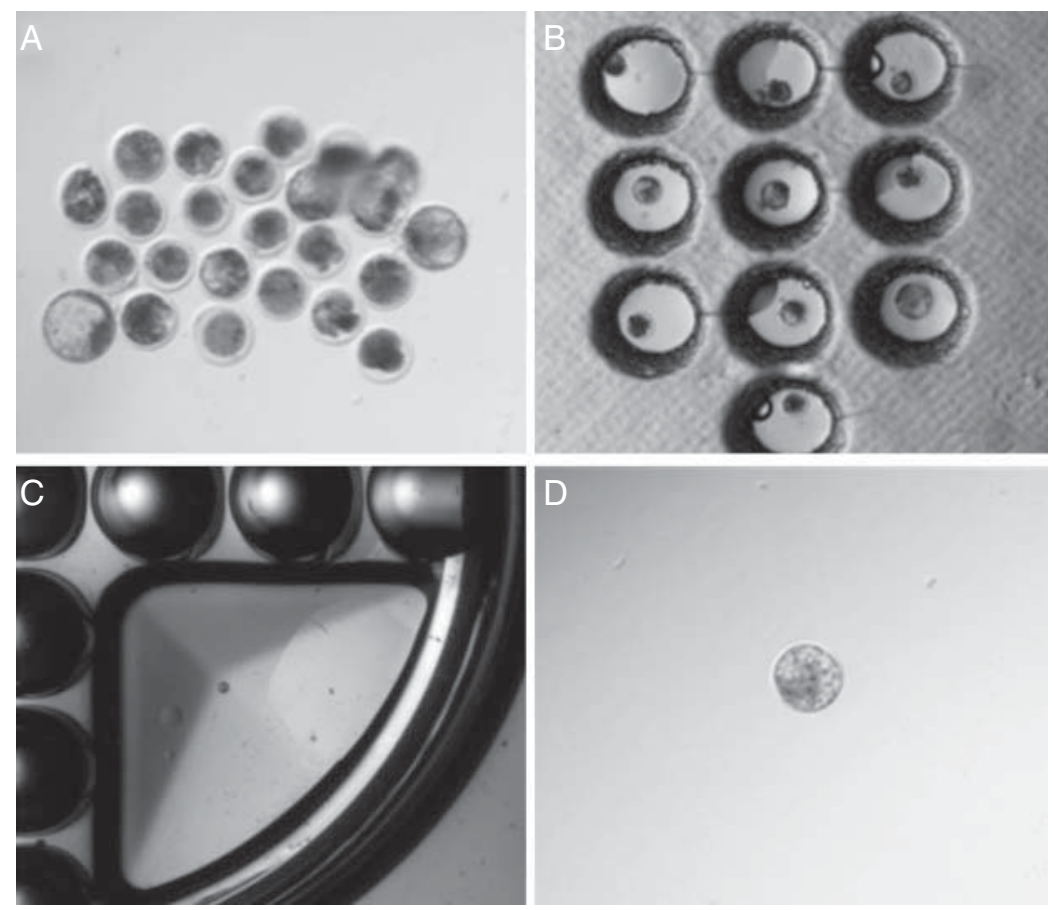

Figure 1 Different culture systems investigated: (A) group culture: inseminated oocytes were cultured in groups of 25 in a $50 \mu \mathrm{l}$ drop of culture medium covered with mineral oil. (B) Group culture in Primo Vision dishes: ten inseminated oocytes were placed in the ten microwells (one embryo per microwell) and covered by a single drop of $30 \mu \mathrm{l}$ culture medium and mineral oil. (C) Group culture in embryo Corral dishes: one embryo was placed in each quadrant of the centre well, so that four inseminated oocytes were cultured as a group, divided by a raster in a drop of $120 \mu$ l culture medium covered by mineral oil. (D) Individual culture: inseminated oocytes were cultured individually in $20 \mu \mathrm{l}$ drops of medium. Seventeen drops were placed in one plate and covered by mineral oil. 
Table 2 Definition of standard and delayed drops in Primo Vision dishes based on the average percentage obtained in the classical group culture system.

\begin{tabular}{llclcr}
\hline $\begin{array}{l}\text { Time post in vitro } \\
\text { insemination }(\mathrm{hpi})\end{array}$ & Embryo stage & $\begin{array}{c}\text { Reference value in } \\
\text { classical group } \\
\text { culture system }(\%)\end{array}$ & \multicolumn{2}{c}{$\begin{array}{c}\text { No. of embryos in droplet which reached the } \\
\text { same stage in a Primo Vision dish }\end{array}$} \\
\hline 45 & Not cleaved & 20.6 & $\Rightarrow$ & Delayed drop & \multicolumn{1}{c}{ Standard drop } \\
\hline 168 & Fast embryos & 18.8 & $\Rightarrow$ & $<2 / 9$ & $\leq 2 / 9$ \\
192 & Blastocyst & 23.1 & $\Rightarrow$ & $<2 / 9$ & $\geq 2 / 9$ \\
\hline
\end{tabular}

Statistics 19 was used, with group and replicate as fixed effects. A Bonferroni's correction was used to correct for the multiple comparison. Significance level was set at a corrected $P$ value $<0.05$. Data concerning the embryo quality such as TCN, ICM ratio, ACR and absolute ICM-number and apoptotic cell number were analysed using a mixed model ANOVA, with group as the fixed effect and replicate as the random effect. Since the data concerning ACR in Experiment 1 were not normally distributed, a log transformation of the data was applied in order to obtain normally distributed (KolmogorovSmirnov test, $P$ value $>0.01$ ) and homogeneous data (Levene test, $P$ value $>0.05)$. Evaluation of the strengths and weaknesses of group culture was carried out retrospectively using the data from Experiment 1. All variables were tested simultaneously using a binary logistic regression model of IBM SPSS Statistics 19, with group and replicate as fixed effects. A $P$ value $<0.05$ was considered statistically significant.

\section{Results}

\section{Experiment 1: comparison of different group culture systems}

\section{Embryo development}

The embryo culture system did not affect the embryo cleavage rate at $45 \mathrm{hpi}$. Similarly, no differences were observed in the proportion of 'fast' (five to eight cell stages) and 'slow' (two to four cell stages) embryos (Table 3). Blastocyst development at $168 \mathrm{hpi}$ was not significantly different between the culture systems. At $192 \mathrm{hpi}$, however, a higher proportion of blastocysts were observed in Primo Vision dishes compared with individually cultured embryos and those cultured in Corral dishes. Blastocyst development at $192 \mathrm{hpi}$ in Primo Vision dishes was not different from that in the classical group culture system. No differences in hatching rate were observed between the culture systems investigated.

\section{Embryo quality}

Embryo quality was evaluated by measuring three parameters simultaneously: i) the $\mathrm{TCN}$; ii) the ICM ratio as the number of cells of the ICM compared with the $\mathrm{TCN}$ and iii) the ACR. Higher TCNs were recorded in blastocysts derived from classical group culture and culture in Primo Vision dishes compared with individual culture and culture in Corral dishes. The ICM ratio was similar for all four groups, although the actual number of ICM cells was higher in embryos cultured in classical group culture or in Primo Vision dishes, compared with Corral dishes or individual culture (GR, 67.6 $\pm 1.93^{\mathrm{a}}$; Primo Vision, $62.8 \pm 1.89^{\mathrm{a}}$; Corral, $50.7 \pm 1.98^{\mathrm{b}}$ and IND, $50.4 \pm 1.51^{\mathrm{b}}$ ). The ACR was higher for all the test groups compared with classical group culture (Fig. 2). However, the actual number of apoptotic cells was higher only in blastocysts cultured in Corral dishes compared with classical group culture $\left(G R, 7.6 \pm 0.26^{\mathrm{ab}}\right.$; Primo Vision, $8.6 \pm 0.32^{\mathrm{a}}$; Corral, $8.9 \pm 0.35^{\mathrm{ac}}$ and IND, $\left.8.0 \pm 0.24^{\mathrm{a}}\right)$.

\section{Efficiency of group culture vs individual culture methods}

In total, blastocyst development was compared for 245 'slow' embryos in Primo Vision dishes and 242 'slow' embryos cultured individually; 75 'fast' embryos cultured in Primo Vision dishes and 77 'fast' embryos cultured individually. The higher rate of blastocyst development at 192 hpi observed in Primo Vision dishes compared with individual culture was due mainly to a significant increase in the development of 'slow'

Table 3 Embryo development in the different culture systems.

\begin{tabular}{|c|c|c|c|c|c|c|}
\hline & \multirow[b]{2}{*}{$n$} & \multicolumn{2}{|c|}{ Cleavage rate at $45 \mathrm{hpi}(\% \pm$ s.D. $)$} & \multicolumn{3}{|c|}{ Blastocyst yield (\% $\%$ S.D.) } \\
\hline & & Fast embryos & Slow embryos & $168 \mathrm{hpi}$ & 192 hpi & Hatching rate $^{a}$ \\
\hline Group & 399 & $18.8 \pm 1.96$ & $60.9 \pm 2.21$ & $23.1 \pm 2.11$ & $34.3 \pm 2.38^{*}$ & $19.7 \pm 3.40$ \\
\hline Primo Vision & 382 & $18.6 \pm 1.99$ & $65.2 \pm 2.06$ & $25.9 \pm 2.24$ & $39.0 \pm 2.22^{* t}$ & $13.4 \pm 2.79$ \\
\hline Corral & 384 & $18.5 \pm 1.98$ & $56.5 \pm 2.53$ & $17.7 \pm 1.95$ & $28.9 \pm 2.31^{*, \neq}$ & $15.3 \pm 3.42$ \\
\hline Individual & 407 & $18.7 \pm 1.93$ & $59.2 \pm 2.44$ & $20.1 \pm 1.99$ & $28.5 \pm 2.24^{*, \neq}$ & $16.4 \pm 3.44$ \\
\hline
\end{tabular}

Data are expressed as mean \pm s.D., differences at $P<0.05$ were considered significant and are marked with different superscript. hpi, hours post in vitro insemination.

${ }^{a}$ Hatching rates are expressed as the percentage of hatching or hatched blastocysts relative to the number of blastocysts at $192 \mathrm{hpi}$. 


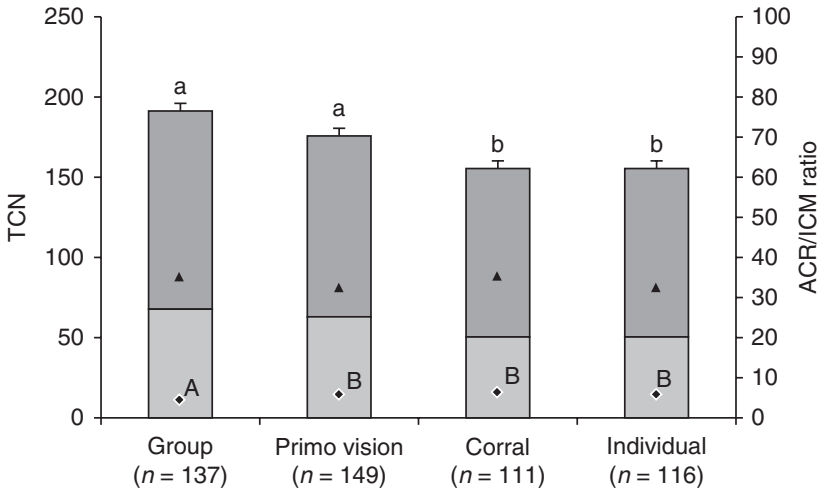

Figure 2 Embryo quality in the different culture systems: total cell number (TCN) as the sum of trophectoderm cells (TE; dark grey) and cells allocated to the ICM (calculated as TCN-TE) (light grey), ICMratio (filled triangle) and apoptotic cell ratio (ACR; filled diamond) of blastocysts collected after $192 \mathrm{hpi}$ and differential apoptotic staining. Embryos were cultured in classical group cultures, Primo Vision dishes, Corral dishes or as individuals; differences between the different culture systems that were significant $(P<0.05)$ are marked with a different superscript.

embryos to blastocyst stage in Primo Vision dishes compared with individual culture (Table 4). No such increase in blastocyst development occurred for 'fast' embryos in Primo Vision dishes compared with individual culture (fig 5).

Depending on their position in the Primo Vision dish, individual embryos could be accompanied by 3, 4, 5, 6 or 8 adjacent embryos, defined as 'neighbours' (Fig. 3). The proportion of 'slow' embryos that reached blastocyst stage at 192 hpi were higher for Primo Vision dishes than for those cultured individually, especially if they were surrounded by four to six neighbours (Fig. 4, part 1). For fast embryos, however, the number of neighbours in Primo Vision dishes did not affect the proportion of embryos that developed into blastocysts (Fig. 5, part 1).

In addition to the analysis of the effect of the number of adjacent embryos, the effect of the developmental stage of the 'neighbours' at different time points was also examined. This involved looking retrospectively at the developmental stage of neighbouring embryos at $45 \mathrm{hpi}$ and recording the percentage that were non-cleaved (unfertilised oocytes or arrested embryos) designated as neighbours with delayed development. We analysed whether the percentage of neighbours with delayed development for embryos cultured in a Primo Vision dish (containing either 0-25, 26-50, 51-75 and 76-100\% non-cleaved or arrested embryos) affected blastocyst development at $192 \mathrm{hpi}$ of both 'slow' and 'fast' embryos vs embryos cultured individually. Blastocyst development of 'slow' embryos was enhanced in Primo Vision dishes compared with individual culture when $<25 \%$ of the neighbouring embryos were non-cleaved at $45 \mathrm{hpi}$. However, when more than $25 \%$ of neighbouring embryos were non-cleaved at $45 \mathrm{hpi}$, development of 'slow' embryos to blastocyst stage was not different from the rate of 'slow' embryos cultured individually (Fig. 4, part 2A). For 'fast' embryos, the proportion of neighbouring non-cleaved embryos did not reduce blastocyst development compared with individually cultured 'fast' embryos (Fig. 5, part 2A).

Using the same approach to analyse the influence of having adjoining embryos showing a standard rate of development (i.e. 'fast' cleaving embryos at 45 hpi and embryos reaching the blastocyst stage at 168 and $192 \mathrm{hpi})$, it transpired that when either $0-25$ or $26-50 \%$ of the neighbours of 'slow' embryos were 'fast' cleavers at $45 \mathrm{hpi}$, a higher rate of blastocyst development by 'slow' embryos compared with their individually cultured counterparts was observed (data not shown). On the contrary, for 'fast' embryos, the proportion of 'fast' cleaving neighbours did not influence the blastocyst development at 192 hpi compared with individual culture (data not shown). The proportion of neighbours of 'slow' embryos that reached the blastocyst stage at 168 and 192 hpi (Fig. 4, parts 2B and C) are likely to be less important; all the categories scored better than individual culture, except for the group of $26-50 \%$ of the neighbours developing into a blastocyst at $192 \mathrm{hpi}$. For 'fast' embryos, on the other hand, no differences were observed between the individually cultured 'fast' embryos vs those cultured in groups; however, a higher number of embryos are needed to confirm these observations (Fig. 5, parts $2 \mathrm{~B}$ and $\mathrm{C}$ ).

Finally, examination of the effect of being in a so-called 'standard' or a 'delayed' drop showed that the number of non-cleaved embryos in Primo Vision dishes was not associated with a reduced rate of blastocyst development in group culture; this was the case for both 'slow' and 'fast' embryos. When a

Table 4 Effect of removal of non-cleaved embryos or regrouping of cleaved embryos on embryo development in Primo Vision dishes.

\begin{tabular}{|c|c|c|c|c|c|c|}
\hline \multirow[b]{2}{*}{ Primo Vision } & \multirow[b]{2}{*}{$n$} & \multicolumn{2}{|c|}{ Cleavage rate at $45 \mathrm{hpi}(\% \pm$ s.D. $)$} & \multicolumn{3}{|c|}{ Blastocyst yield (\% $\%$ S.D.) } \\
\hline & & Fast embryos & Slow embryos & $168 \mathrm{hpi}$ & $192 \mathrm{hpi}$ & Hatching rate $^{\mathrm{a}}$ \\
\hline CTRL & 225 & $20.0 \pm 2.67$ & $60.0 \pm 3.27$ & $18.7 \pm 2.60$ & $31.6 \pm 3.10$ & $11.3 \pm 3.76^{*}$ \\
\hline NC removed & 225 & $16.0 \pm 2.44$ & $60.4 \pm 3.26$ & $15.1 \pm 2.39$ & $29.3 \pm 3.03$ & $9.1 \pm 3.54^{*}$ \\
\hline ReGR & 179 & $18.4 \pm 2.90$ & $63.7 \pm 3.59$ & $20.1 \pm 2.99$ & $29.6 \pm 3.41$ & $30.2 \pm 6.31^{\dagger}$ \\
\hline
\end{tabular}

Data are expressed as mean \pm s.D., differences at $P<0.05$ were considered significant and are marked with different superscript. hpi, hours post in vitro insemination; CTRL, control; NC removed, removal of non-cleaved embryos; ReGR, removal of non-cleaved embryos with subsequent regrouping of the cleaved ones.

${ }^{a}$ Hatching rates are expressed as the percentage of hatching or hatched blastocysts relative to the number of blastocysts at $192 \mathrm{hpi}$. 


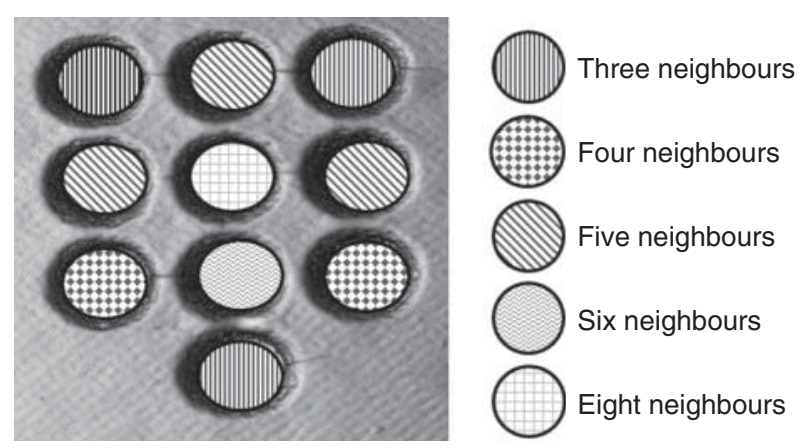

Figure 3 Presentation of the number of neighbours in Primo Vision: depending on their position in the Primo Vision dish, embryos could have 3, 4, 5, 6 or 8 neighbours (adjacent embryos).

particular embryo was a 'slow' embryo, it benefited from group culture in Primo Vision dishes, as reflected by a higher rate of blastocyst development at $192 \mathrm{hpi}$ compared with individually cultured 'slow' embryos. This was not dependent on the number of non-cleaved embryos at 45 hpi (Fig. 4, part 3D). By contrast, 'fast' embryos did not show a higher rate of blastocyst development at $192 \mathrm{hpi}$ following group vs individual culture, regardless of whether the embryo resided in a drop with two or more non-cleaved embryos (Fig. 5, part 3D). The number of 'fast' embryos in the drop at $45 \mathrm{hpi}$ did not influence the rate of blastocyst development of either 'slow' or 'fast' embryos in group culture compared with their individually cultured counterparts (data not shown). The 'slow' embryos benefited from the vicinity of more than two blastocysts at $168 \mathrm{hpi}$ and this was even more pronounced at $192 \mathrm{hpi}$ (Fig. 4, parts 3E and F). For 'fast' embryos, however, if less than three of the embryos in the group had developed into a blastocyst at $192 \mathrm{hpi}$, the chance of that particular embryo becoming a blastocyst compared with individually cultured 'fast' embryos was significantly reduced (Fig. 5, part 3F).

\section{Experiment 2: effect of non-cleaved embryos in group culture}

Removal of non-cleaved embryos from the culture, with or without regrouping the cleaved embryos afterwards, did not influence embryo cleavage at 45 hpi. Similarly, no differences were observed in the proportions of 'fast' (five to eight cells) and 'slow' embryos (two to four cells; Table 4). Blastocyst development at 168 and $192 \mathrm{hpi}$ was likewise not affected. However, a higher hatching rate was observed at $192 \mathrm{hpi}$ when the cleaved embryos were regrouped after removal of the non-cleaved embryos compared with the control. No differences in blastocyst quality could be observed between the groups in terms of TCN, ICM-ratio or ACR (Fig. 6), absolute ICM-number (CTRL, 67.1 \pm 2.61 ; NC removed, $56.0 \pm 2.57$ and $\operatorname{ReGR}, 66.7 \pm 2.88)$ and apoptotic cell number (CTRL, 6.6 $\pm 0.42 ; \mathrm{NC}$ removed, $6.8 \pm 0.41$ and ReGR, 7.2 \pm 0.45$)$.

\section{Discussion}

In this study, two commercially available group culture systems, Corral dishes and Primo Vision dishes, were compared using bovine embryos and following the development of individual embryos. The results clearly showed that Primo Vision dishes were advantageous compared with culture in Corral dishes or culture of individual embryos in terms of both the development and quality of the embryos. Moreover, embryo development in Primo Vision dishes was not different from that in a classical group culture system, thereby allowing the conclusion that Primo Vision dishes combine the benefits of both group and individual culture of mammalian embryos.

There are several possible explanations for the improved embryo development in Primo Vision as compared with Corral culture dishes. First is the higher density of embryos in the Primo Vision dishes, as given in Table 1. For bovine and mouse embryos, an embryo density of one embryo per $2 \mu \mathrm{l}$ medium has been described as the optimum density to maximise the effects of autocrine factors (Kato \& Tsunoda 1994, Palasz \& Thundathil 1998). Second, the smaller inter-embryo distance in Primo Vision vs Corral dishes is advantageous (Table 1). Gopichandran \& Leese (2006) described an inter-embryo distance of $165 \mu \mathrm{m}$ as being optimal for blastocyst formation of bovine embryos. Third, the microwells in the Primo Vision dish contribute to more efficient autocrine communication between embryos. Group culture with individual follow-up in microwells was shown to significantly improve the developmental potential of such group-cultured embryos (Vajta et al. 2000, Hoelker et al. 2009). This improvement was not strictly related to the dish used since individual culture of bovine embryos in Primo Vision dishes at a rate of one embryo per $30 \mu \mathrm{l}$ medium did not result in blastocyst development (data not shown).

It is generally accepted that WOW culture provides a good compromise between the controversial needs of large volume (nutrition and dilution of toxic components) and small volume (concentration of autocrine factors) of a culture medium. In a recent study, which used a mathematical model to calculate the concentration of secreted materials surrounding the embryo, Matsuura (2014) has demonstrated that, when embryos are cultured in microwells, a higher concentration of secreted macromolecules (autocrine factors) and better diffusion of detrimental small molecules, such as ammonia, can be expected compared with the situation in conventional culture systems. In microwells, the direction of such diffusion is restricted to the vertical dimension, whereas in conventional systems diffusion is facilitated both vertically and horizontally, a reduced concentration of autocrine factors around the embryos can be expected. 


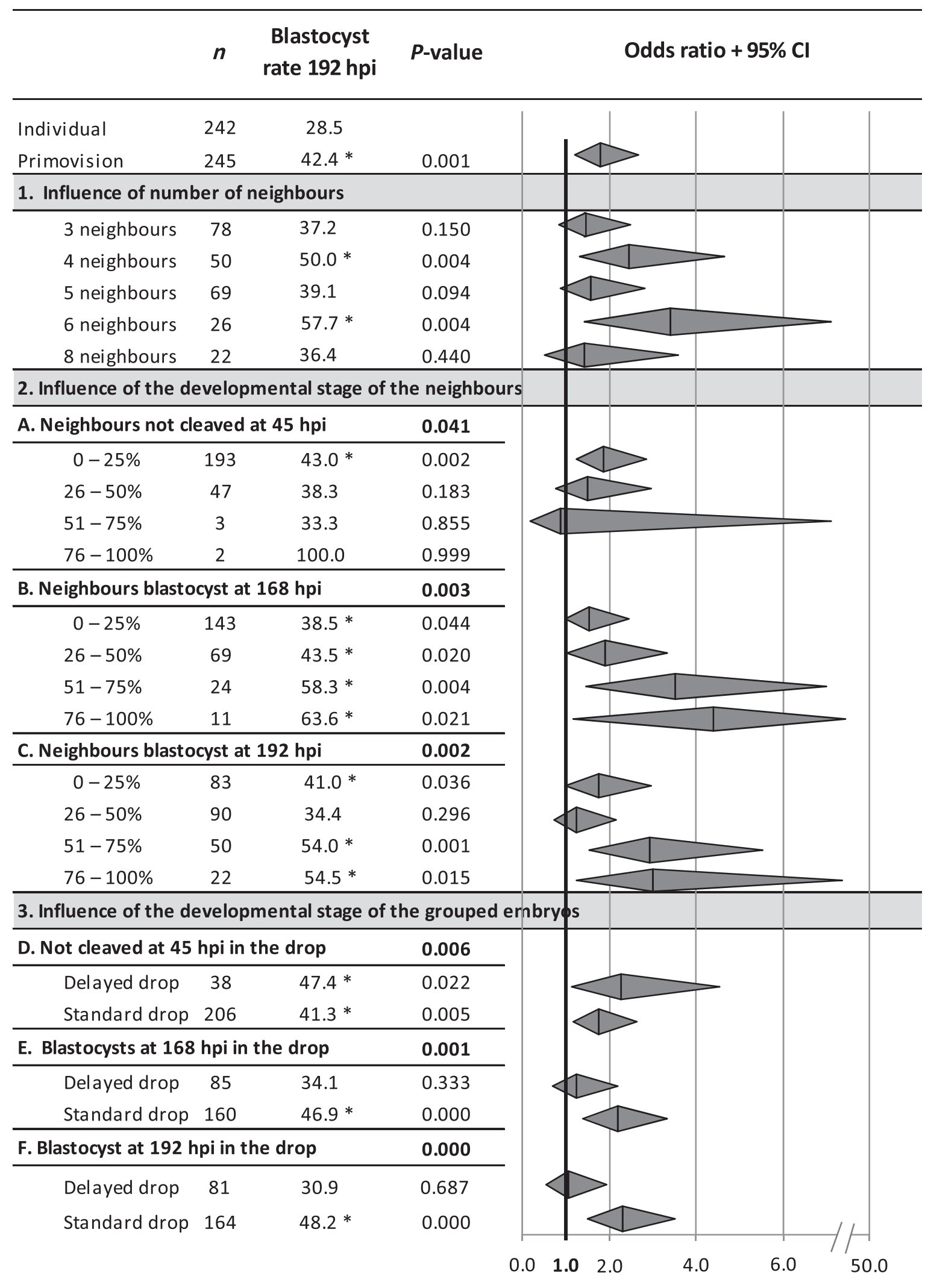

Figure 4 Influence of the number of neighbours and developmental stage of the neighbours and the grouped embryos on the blastocyst development of slow embryos (two to four cells at $45 \mathrm{hpi}$ ) compared with their individually cultured counterparts. Differences between the groups and the individually cultured embryos that were significant $(P<0.05)$ are marked with an asterisk. 


\section{n $\quad \begin{gathered}\text { Blastocyst } \\ \text { rate } 192 \mathrm{hpi}\end{gathered}$-value Odds ratio $+95 \% \mathrm{Cl}$}

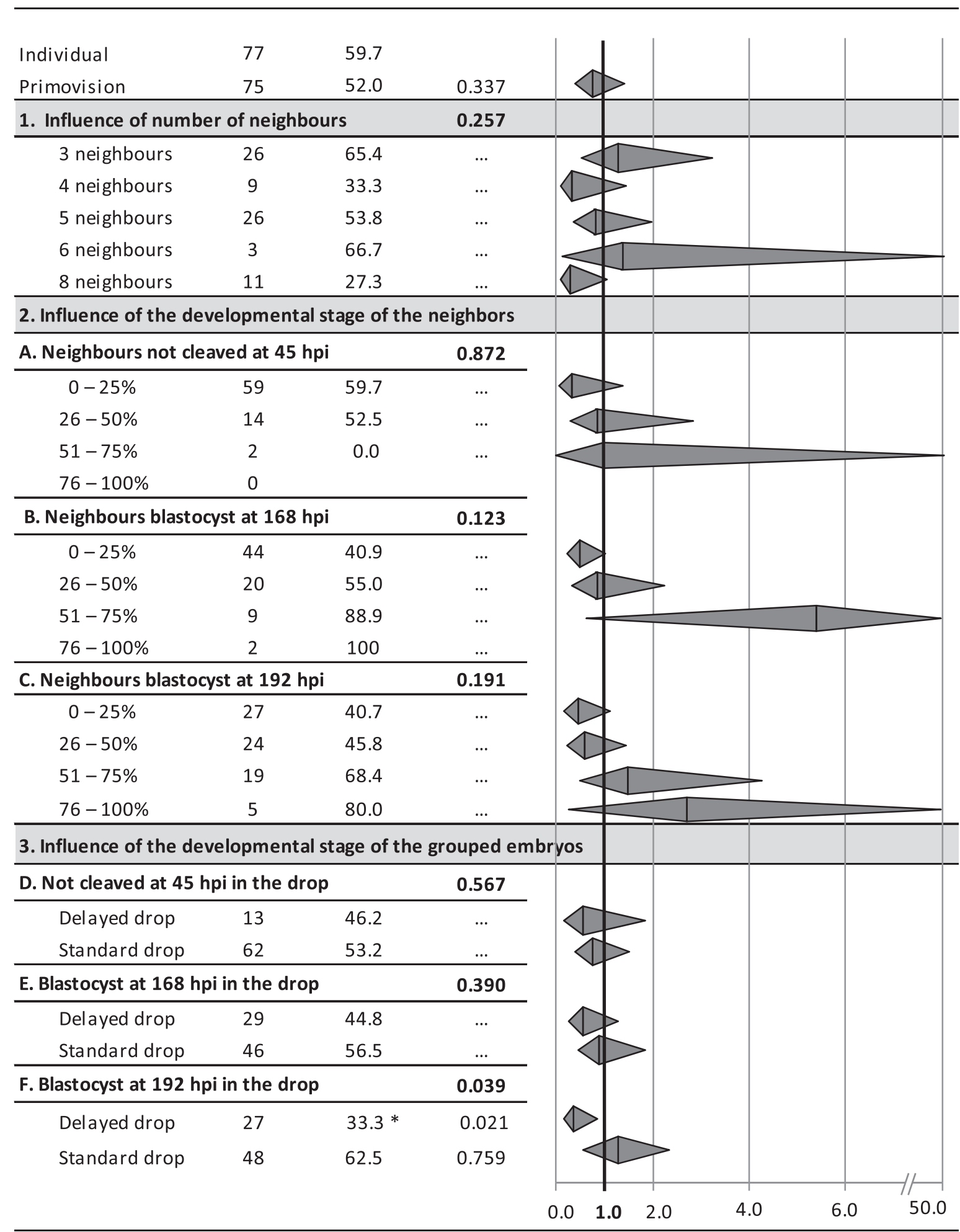

Figure 5 Influence of the number of neighbours and developmental stage of the neighbours and the grouped embryos on the blastocyst development of fast embryos (five to eight cells at $45 \mathrm{hpi}$ ) compared with their individually cultured counterparts. Differences between the groups and the individually cultured embryos that were significant $(P<0.05)$ are marked with an asterisk. 


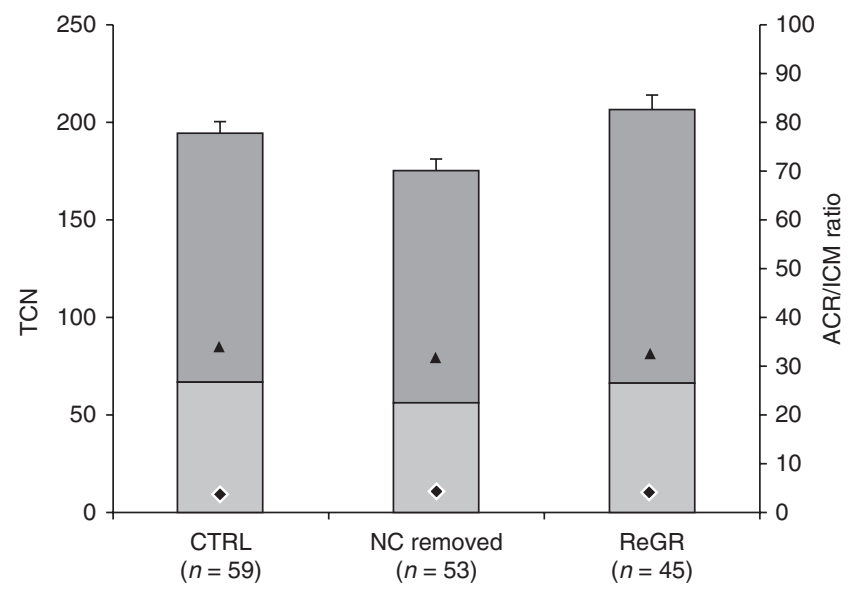

Figure 6 Effect of removal of non-cleaved embryos or regrouping of cleaved embryos on embryo quality in Primo Vision dishes: total cell number (TCN) as the sum of trophectoderm cells (TE; dark grey) and cells allocated to the ICM (calculated as TCN-TE) (light grey), ICM-ratio (filled triangle) and apoptotic cell ratio (ACR; filled diamond) of blastocysts collected at $192 \mathrm{hpi}$ after differential apoptotic staining. Embryos were cultured in Primo Vision dishes (CTRL), with removal of the non-cleaved embryos (NC removed) and subsequent regrouping (ReGR).

Although group culture is used extensively for mammalian embryos, there are no data available on the possibly negative effects of arrested or delayed embryos being cultured in the same drop as a normal embryo. Nor is there any evidence of which embryos benefit most from the group culture effect. In this study, however, it was possible to demonstrate that blastocyst development in Primo Vision dishes is not different from classical group culture, but is significantly better than individual culture. This beneficial outcome resulted mainly from a higher rate of blastocyst development from 'slow' embryos which did much better in the Primo Vision dish than in individual culture. Concern could arise due to more low-grade embryos developing to the blastocyst stage thereby implying that such blastocysts could be of lower quality. As embryo development in vitro is slower than that in vivo (Van Soom et al. 1997), 'fast' developing embryos have, historically, been preferred instead of 'slow' embryos. However, recent data from mice surprisingly cast doubt on this hypothesis. Namely 'slow' embryos are more in vivolike with respect to genomic imprinting and expression of metabolic markers. 'Fast' embryos, on the other hand, show a loss of genomic imprinting, perhaps because the kinetics of high cleavage rates leaves insufficient time to ensure the correct passage of epigenetic information (Velker et al. 2012).

When studying the influence of the 'neighbours', it appears that the number of these does not hamper an embryo's potential to reach the blastocyst stage. This is in agreement with the previous study of Stokes et al. (2005), who could not demonstrate a significant effect on blastocyst development of a porcine embryo's position in a $4 \times 4$ grid. More importantly, it appears to be the developmental stage of the neighbours, and more specifically their development after $45 \mathrm{hpi}$, which positively affects the outcome of a particular embryo. A markedly higher percentage of 'slow' embryos had developed into blastocysts by $192 \mathrm{hpi}$ if they had been surrounded by many embryos that had also developed into blastocysts at 168 or $192 \mathrm{hpi}$, when compared with 'slow' embryos cultured individually. This supportive effect of group culture is presumably related to the production of autocrine factors, although the identity of most of these autocrine embryonic factors remains unknown.

For some growth factors, such as basic fibroblast growth factor (bFGF), insulin-like growth factor 1 (IGF1) and IGF2, interferon, platelet-derived growth factor alfa and transforming growth factor beta (TGF $\beta$ ), it has been demonstrated that the appropriate mRNA is expressed in bovine preimplantation embryos (Watson et al. 1992). However, no studies have yet confirmed the presence of these growth factor proteins or of other potential autocrine factors, in culture medium conditioned by bovine embryos. To date only stress-related proteins have been identified (Wydooghe et al. 2011b). In contrast, some secreted factors have already been identified for cultured human embryos and these can be used as biomarkers for embryo selection. The phospholipid, platelet activation factor (PAF), was the first molecule identified in human embryo-conditioned medium, and now it has also been demonstrated in the culture medium of murine, sheep, rabbit and hamster embryos where it stimulates embryo metabolism, cellcycle progression and embryo viability (reviewed by $\mathrm{O}^{\prime}$ Neill (2005)). Furthermore, the level of PAF produced by human preimplantation embryos in culture medium is positively correlated with pregnancy rate in women (Roudebush et al. 2002). Other proteins secreted by human embryos into the culture medium include soluble human leucocyte antigen-G (Noci et al. 2005, Sher et al. 2005) and ubiquitin (Katz-Jaffe et al. 2006), the latter factor being correlated with on-going blastocyst development. Ubiquitin is a component of the ubiquitin-dependent proteasome system which is involved in a number of physiological processes in somatic cells, including transcription (reviewed by Geng et al. (2012)) and apoptosis (reviewed by Jesenberger \& Jentsch (2002)). Further research is needed to determine its importance as an autocrine factor, as well as to identify other autocrine factors in embryo culture medium.

In this study we showed that bovine embryos that did had not cleaved by $45 \mathrm{hpi}$ did not negatively affect the potential of embryos in the same group to become blastocysts at $192 \mathrm{hpi}$, irrespective of whether the embryo in question was a 'fast' or a 'slow' one. Furthermore, removing the non-cleaved embryos did not increase blastocyst development nor blastocyst quality; although regrouping the cleaved embryos after uncleaved embryo removal was able to increase their 
hatching rate. Neira et al. (2010) have shown that some of the growth factors of which Watson et al. (1992) identified the mRNA in bovine embryos can facilitate the hatching of bovine blastocysts when added to the culture medium (e.g. IGF1, bFGF and TGF $\beta$ ).

Care should be taken when 'fast' embryos are group cultured and only a few of the surrounding embryos reach the blastocyst stage as this could compromise blastocyst development compared with individually cultured 'fast' embryos. 'Fast' developing embryos may be more sensitive to the negative effects of metabolic by-products such as ammonia or lactate or to depletion of necessary substrates by other embryos (Reed et al. 2011). Our present findings indicate that removing 'fast' embryos from a Primo Vision dish for subsequent culture individually may be beneficial whereas 'slow' embryos should remain in the group culture to yield a bigger number of blastocysts.

In summary, our results demonstrated that culturing bovine embryos in small groups in Primo Vision dishes provides an excellent system that combines the benefits of group and individual culture, leads to a similar rate of embryo development compared with classical group culture and gives better embryo development and quality than individual culture or culture in Corral dishes. When more than one-third of neighbouring embryos developed into blastocysts at $192 \mathrm{hpi}$, 'slow' embryos had a higher chance of becoming a blastocyst in comparison with individual culture (48.2\%; OR: 2.3$)$. Whereas, when $<30 \%$ of the neighbouring embryos reached the blastocysts stage, blastulation of 'fast' embryos was less efficient than in individual culture (33.3\%; OR: 0.3). The study also showed that the net outcome of group culture of bovine embryos in Primo Vision dishes is advantageous compared with individual culture, giving a higher blastocyst development rate and improved blastocyst quality. Further research is needed to extrapolate these observations to human embryos.

\section{Declaration of interest}

The authors declare that there is no conflict of interest that could be perceived as prejudicing the impartiality of the research reported.

\section{Funding}

This study was supported by a grant from 'Agentschap voor Innovatie door Wetenschap en Technologie' (grant number, 101122) and Research Foundation - Flanders (grant number, G.0210.09). P De Sutter holds a fundamental clinical research mandate by the Flemish Foundation for Scientific Research (FWO Vlaanderen).

\section{Acknowledgements}

The authors wish to thank Isabel Lemahieu and Petra Van Damme for their excellent technical assistance.

\section{References}

Canseco RS, Sparks AE, Pearson RE \& Gwazdauskas FC 1992 Embryo density and medium volume effects on early murine embryo development. Journal of Assisted Reproduction and Genetics 9 454-457. (doi:10.1007/BF01204051)

Donnay I, VanLangendonckt A, Auquier P, Grisart B, Vansteenbrugge A, Massip A \& Dessy F 1997 Effects of co-culture and embryo number on the in vitro development of bovine embryos. Theriogenology 47 1549-1561. (doi:10.1016/S0093-691X(97)00160-X)

Ebner T, Shebl O, Moser M, Mayer RB, Arzt W \& Tews G 2010 Group culture of human zygotes is superior to individual culture in terms of blastulation, implantation and life birth. Reproductive Biomedicine Online 21 762-768. (doi:10.1016/j.rbmo.2010.06.038)

Fischer-Brown A, Monson R, Parrish J \& Rutledge J 2002 Cell allocation in bovine embryos cultured in two media under two oxygen concentrations. Zygote 10 341-348. (doi:10.1017/S0967199402004082)

Geng FQ, Wenzel S \& Tansey WP 2012 Ubiquitin and proteasomes in transcription. Annual Review of Biochemistry 81 177-201. (doi:10.1146/ annurev-biochem-052110-120012)

Goovaerts IG, Leroy JL, Van Soom A, De Clercq JB, Andries S \& Bols PE 2009 Effect of cumulus cell coculture and oxygen tension on the in vitro developmental competence of bovine zygotes cultured singly. Theriogenology 71 729-738. (doi:10.1016/j.theriogenology.2008.09. 038)

Goovaerts IG, Leroy JL, Langbeen A, Jorssen EP, Bosmans E \& Bols PE 2012 Unravelling the needs of singly in vitro-produced bovine embryos: from cumulus cell co-culture to semi-defined, oil-free culture conditions. Reproduction, Fertility, and Development 24 1084-1092. (doi:10.1071/ RD11286)

Gopichandran N \& Leese HJ 2006 The effect of paracrine/autocrine interactions on the in vitro culture of bovine preimplantation embryos. Reproduction 131 269-277. (doi:10.1530/rep.1.00677)

Hoelker MR, Rings F, Lund Q, Ghanem N, Phatsara C, Griese J, Schellander K \& Tesfaye D 2009 Effect of the microenvironment and embryo density on developmental characteristics and gene expression profile of bovine preimplantative embryos cultured in vitro. Reproduction 137 415-425. (doi:10.1530/REP-08-0370)

Jesenberger V \& Jentsch S 2002 Deadly encounter: ubiquitin meets apoptosis. Nature Reviews. Molecular Cell Biology 3 112-121. (doi:10. 1038/nrm731)

Kato Y \& Tsunoda Y 1994 Effects of the culture density of mouse zygotes on the development in-vitro and in-vivo. Theriogenology 41 1315-1322. (doi:10.1016/0093-691X(94)90490-A)

Katz-Jaffe MG, Schoolcraft WB \& Gardner DK 2006 Analysis of protein expression (secretome) by human and mouse preimplantation embryos. Fertility and Sterility 86 678-685. (doi:10.1016/j.fertnstert.2006.05.022)

Lane M \& Gardner DK 1992 Effect of incubation volume and embryo density on the development and viability of mouse embryos in vitro. Human Reproduction 7 558-562.

Matsuura K 2014 Numerical calculations for diffusion effects in the Well-of-the-Well culture system for mammalian embryos. Reproduction, Fertility, and Development 26 742-751. (doi:10.1071/RD13025)

Neira JA, Teinturier D, Peñab MA \& Martal J 2010 Effect of the association of IGF-I, IGF-II, bFGF, TGF- $\beta 1$, GM-CSF, and LIF on the development of bovine embryos produced in vitro. Theriogenology 73 595-604. (doi:10.1016/j.theriogenology.2009.10.015)

Noci I, Fuzzi B, Rizzo R, Melchiorri L, Criscuoli L, Dabizzi S, Biagiotti R, Pellegrini S, Menicucci A \& Baricordi OR 2005 Embryonic soluble HLA-G as a marker of developmental potential in embryos. Human Reproduction 20 138-146. (doi:10.1093/humrep/deh572)

O'Doherty EM, Wade MG, Hill JL \& Boland MP 1997 Effects of culturing bovine oocytes either singly or in groups on development to blastocysts. Theriogenology 48 161-169. (doi:10.1016/S0093-691X(97)00199-4)

O'Neill C 2005 The role of paf in embryo physiology. Human Reproduction Update 11 215-228. (doi:10.1093/humupd/dmi003)

O'Neill C 2008 The potential roles for embryotrophic ligands in preimplantation embryo development. Human Reproduction Update $\mathbf{1 4}$ 275-288. (doi:10.1093/humupd/dmn002)

Palasz AT \& Thundathil J 1998 The effect of volume of culture medium and embryo density on in vitro development of bovine embryos. Theriogenology 49 212-212. (doi:10.1016/S0093-691X(98)90565-9) 
Paria BC \& Dey SK 1990 Preimplantation embryo development in vitro: cooperative interactions among embryos and role of growth-factors. PNAS 87 4756-4760. (doi:10.1073/pnas.87.12.4756)

Rebollar-Lazaro I \& Matson P 2010 The culture of human cleavage stage embryos alone or in groups: effect upon blastocyst utilization rates and implantation. Reproductive Biology 10 227-234. (doi:10.1016/S1642431X(12)60042-4)

Reed ML, Woodward BJ \& Swain JE 2011 Single or group culture of mammalian embryos: the verdict of the literature. Journal of Reproductive and Stem Cell Biotechnology 2 77-87.

Rijnders PM \& Jansen CA 1999 Influence of group culture and culture volume on the formation of human blastocysts: a prospective randomized study. Human Reproduction 14 2333-2337. (doi:10.1093/ humrep/14.9.2333)

Roudebush WE, Wininger JD, Jones AE, Wright G, Toledo AA, Kort HI, Massey JB \& Shapiro DB 2002 Embryonic platelet-activating factor: an indicator of embryo viability. Human Reproduction 17 1306-1310. (doi:10.1093/humrep/17.5.1306)

Sagirkaya H, Misirlioglu M, Kaya A, First NL, Parrish JJ \& Memili E 2007 Developmental potential of bovine oocytes cultured in different maturation and culture conditions. Animal Reproduction Science 101 225-240. (doi:10.1016/j.anireprosci.2006.09.016)

Salahuddin S, Ookutsu S, Goto K, Nakanishi Y \& Nagata Y 1995 Effects of embryo density and coculture of unfertilized oocytes on embryonic development of in vitro fertilized mouse embryos. Human Reproduction 10 2382-2385. (doi:10.1093/oxfordjournals.humrep.a136303)

Sher G, Keskintepe L, Fisch JD, Acacio BA, Ahlering P, Batzofin J \& Ginsburg M 2005 Soluble human leukocyte antigen G expression in phase I culture media at 46 hours after fertilization predicts pregnancy and implantation from day 3 embryo transfer. Fertility and Sterility $\mathbf{8 3}$ 1410-1413. (doi:10.1016/j.fertnstert.2004.11.061)

Spindler RE \& Wildt DE 2002 Quality and age of companion felid embryos modulate enhanced development by group culture. Biology of Reproduction 66 167-173. (doi:10.1095/biolreprod66.1.167)

Spyropoulou I, Karamalegos C \& Bolton VN 1999 A prospective randomized study comparing the outcome of in-vitro fertilization and embryo transfer following culture of human embryos individually or in groups before embryo transfer on day 2. Human Reproduction 14 76-79. (doi:10.1093/humrep/14.1.76)

Stokes PJ, Abeydeera LR \& Leese HJ 2005 Development of porcine embryos in vivo and in vitro; evidence for embryo 'cross talk' in vitro. Developmental Biology 284 62-71. (doi:10.1016/j.ydbio.2005.05.001)

Tao T, Robichaud A, Mercier J \& Ouellette R 2013 Influence of group embryo culture strategies on the blastocyst development and pregnancy outcome. Journal of Assisted Reproduction and Genetics 30 63-68. (doi:10.1007/s10815-012-9892-x)

Thys M, Vandaele L, Morrell JM, Mestach J, Van Soom A, Hoogewijs M \& Rodriguez-Martinez H 2009 In vitro fertilizing capacity of frozen-thawed bull spermatozoa selected by single-layer (glycidoxypropyltrimethoxysilane) silane-coated silica colloidal centrifugation. Reproduction in Domestic Animals 44 390-394. (doi:10.1111/j.1439-0531.2008. 01081.x)

Vajta G, Peura TT, Holm P, Paldi K, Greve T, Trounson AO \& Callesen H 2000 New method for culture of zona-included or zona-free embryos: the Well of the Well (WOW) system. Molecular Reproduction and Development 55 256-264. (doi:10.1002/(SICl)1098-2795(200003) $<55: 3<256:$ :AID-MRD3 > 3.0.CO;2-7)

Vandaele L, Mateusen B, Maes DG, de Kruif A \& van Soom A 2007 Temporal detection of caspase- 3 and- 7 in bovine in vitro produced embryos of different developmental capacity. Reproduction 133 709-718. (doi:10.1530/REP-06-0109)

Van Soom A, Boerjan ML, Bols PE, Vanroose G, Lein A, Coryn M \& deKruif A 1997 Timing of compaction and inner cell allocation in bovine embryos produced in vivo after superovulation. Biology of Reproduction 57 1041-1049. (doi:10.1095/biolreprod57.5.1041)

Velker BA, Denomme MM \& Mann MR 2012 Loss of genomic imprinting in mouse embryos with fast rates of preimplantation development in culture. Biology of Reproduction 86 1-16. (doi:10.1095/biolreprod.111. 096602)

Watson AJ, Hogan A, Hahnel A, Wiemer KE \& Schultz GA 1992 Expression of growth-factor ligand and receptor genes in the preimplantation embryo. Molecular Reproduction and Development 31 87-95. (doi:10.1002/mrd.1080310202)

Wydooghe E, Vandaele L, Beek J, Favoreel H, Heindryckx B, De Sutter P \& Van Soom A 2011 a Differential apoptotic staining of mammalian blastocysts based on double immunofluorescent CDX2 and active caspase-3 staining. Analytical Biochemistry 416 228-230. (doi:10.1016/j.ab.2011. 05.033)

Wydooghe E, Heras S, Vandaele L, Van Steendam K, Dewulf J \& Van Soom A 2011b Pitfalls and promises in revealing the secretome of bovine preimplantation embryos. Proceedings of the 4th General Meeting of GEMINI, Gijon, Spain 83.

Wydooghe E, Heras S, Dewulf J, Piepers S, Van den Abbeel E, De Sutter P, Vandaele L \& Van Soom A 2014 Replacing serum in culture medium with albumin and insulin, transferrin and selenium is the key to successful bovine embryo development in individual culture. Reproduction, Fertility, and Development 26 717-724. (doi:10.1071/ RD13043)

Received 18 April 2014

First decision 5 June 2014

Revised manuscript received 29 July 2014

Accepted 7 August 2014 